\title{
Parent and Child Usual Source of Care and Children's Receipt of Health Care Services
}

Jennifer E. DeVoe, MD, DPbil ${ }^{1}$

Carrie J. Tillotson, $M P H^{1}$

Lorraine S. Wallace, $P b D^{2}$

Heather Angier, $M P H^{1}$

Matthew J. Carlson, $\mathrm{PhD}^{3}$

Rachel Gold, $\mathrm{PbD}, \mathrm{MPH}^{4}$

'Department of Family Medicine, Oregon Health \& Science University, Portland, Oregon

${ }^{2}$ Department of Family Medicine, The Ohio State University, Columbus, Ohio

${ }^{3}$ Portland State University, Portland, Oregon

${ }^{4}$ Kaiser Permanente Northwest Center for Health Research, Portland, Oregon

Conflicts of interest: authors report none.

\section{CORRESPONDING AUTHOR}

Jennifer E. DeVoe, MD, DPhil

Oregon Health \& Science University

Department of Family Medicine

3181 Sam Jackson Park Rd, Mailcode: FM

Portland, OR 97239

devoej@ohsu.edu

\begin{abstract}
PURPOSE In the United States, children who have a usual source of care (USC) have better access to health care than those who do not, but little is known about how parental USC affects children's access. We examined the association between child and parent USC patterns and children's access to health care services.
\end{abstract}

METHODS We undertook a secondary analysis of nationally representative, crosssectional data from children participating in the 2002-2007 Medical Expenditure Panel Survey $(n=56,302)$. We assessed 10 outcome measures: insurance coverage gaps, no doctor visits in the past year, less than yearly dental visits, unmet medical and prescription needs, delayed care, problems getting care, and unmet preventive counseling needs regarding healthy eating, regular exercise, car safety devices, and bicycle helmets.

RESULTS Among children, $78.6 \%$ had a USC and at least 1 parent with a USC, whereas $12.4 \%$ had a USC but no parent USC. Children with a USC but no parent USC had a higher likelihood of several unmet needs, including an insurance coverage gap (adjusted risk ratio [aRR] 1.33; 95\% confidence interval [CI], 1.21-1.47), an unmet medical or prescription need (aRR 1.70; $95 \% \mathrm{Cl} 1.09-2.65$ ), and no yearly dental visits (aRR 1.12; 95\% Cl 1.06-1.18), compared with children with a USC whose parent(s) had a USC.

CONCLUSIONS Among children with a USC, having no parent USC was associated with a higher likelihood of reporting unmet needs when compared with children whose parent(s) had a USC. Policy reforms should ensure access to a USC for all family members.

Ann Fam Med 2011;9:504-513. doi:10.1370/afm.1300.

\section{INTRODUCTION}

7 he association between having a usual source of care (USC) and improved access to health care services is well established in both adult and child populations. ${ }^{1-10}$ Children with a USC have more consistent access to health care services, , $^{2,11-15}$ which likely contributes to better overall health outcomes. ${ }^{16,17}$ According to recent estimates, however, approximately $19 \%$ of adults and nearly $10 \%$ of children in the United States do not have a USC. Safety-net services are oversubscribed, and the availability of primary care is widely disparate, ${ }^{18}$ leaving many families with few options for maintaining a relationship with a USC.

Although previous research clearly supports the importance of the relationship between a child having a stable USC and that individual child's access to and utilization of recommended care, ${ }_{1}^{1-10,19}$ little is known about whether a child's receipt of health care is associated with his or her parents' USC status. In fact, to our knowledge, no previous studies describe child-parent USC patterns or measure how parental USC status affects a child's access to health care services. Further, the effect a child's USC status has on access to health care has been documented without accounting for parental USC status.

It is known that children with health insurance have higher odds of 
unmet health care needs if their parents are uninsured. ${ }^{20-23}$ In addition, child health care service utilization patterns are highly associated with maternal utilization patterns. ${ }^{24,25}$ These relationships underscore the need to explore other possible mechanisms through which parental factors affect children's access to care. Specifically, this study examines the association between child-parent USC patterns and children's access to health care services. Our central research question was whether a child's access to health care services varies in association with whether at least 1 parent has a USC. We hypothesized that children of parents without a USC would have higher rates of unmet health care needs when compared with children whose parents have a USC, independent of the child's USC status.

\section{METHODS}

This study was a secondary analysis of data from the Medical Expenditure Panel Survey-Household Component (MEPS-HC). ${ }^{26}$ MEPS-HC collects data from a subsample of households from the National Health Interview Survey in the United States and utilizes a stratified and clustered random sample with weights that produce nationally representative estimates for the civilian, noninstitutionalized population. ${ }^{27-29}$

MEPS data are reported in yearly files, combining data from 2 overlapping panels for each year (eg, data for 2002 combines the overlapping panels of 2001-2002 and 2002-2003); the MEPS design and methodology are reported elsewhere. ${ }^{26-29}$ Each year of data constitutes a nationally representative sample, and pooling the data produces average annual estimates. We combined data from 2002-2007 and included all children aged 17 years and younger with positive full-year weights who had at least 1 parent residing in the same household, and for whom both the child's and at least 1 parent's USC status could be ascertained ( $n=56,302)$, weighted to a population of nearly 70.9 million children.

We linked each child to 1 or both parents and then constructed child-parent USC variables ( $\mathrm{n}=17,612$ linked to 1 parent $_{i} \mathrm{n}=38,690$ linked to 2 parents). This linkage is possible for biological parents, adopted parents, and step-parents; MEPS does not include similar variables for linking foster parents or nonparent guardians. ${ }^{30}$ The sample size of 56,302 children excludes 2,049 children with no parent identified in the household and an additional 437 children for whom USC status could not be reliably ascertained for the child or parent(s).

\section{Dependent Variables}

Asking parents (or caregivers) about their perceptions of a child's unmet need for care is one of the most common ways to assess unmet need..$^{31}$ Accordingly, we used
MEPS-HC items that pertained to parent-reported unmet health care needs to construct the following 6 unmet need variables: (1) insurance coverage gaps during the year, (2) no doctor visits in the past year, (3) less than yearly dental visits, (4) unmet medical and prescription needs, (5) delayed care, and (6) problems getting care. Yearly doctor visits were chosen because the American Academy of Pediatrics recommends yearly health care visits up to 21 years. ${ }^{32}$ For child dental care, the American Academy of Pediatric Dentistry recommends that yearly dental visits begin at the time of the first tooth and no later than 12 months of age. ${ }^{33}$ The delayed care variable combined MEPS-HC parental reports that a child did not always get care as soon as was wanted for an illness, injury, or condition that needed care right away and that a child did not always get an appointment for routine health care as soon as was wanted. The variable pertaining to problems getting care combined 3 MEPS-HC questions regarding a child having problems obtaining needed medical care, tests, prescriptions, referrals, or other treatments.

Because receipt of age-appropriate preventive services has an important impact on long-term health, ${ }^{34}$ we also constructed 4 composite outcomes pertaining to whether a child had not received anticipatory guidance regarding the importance of healthy eating, regular exercise, use of car safety devices (ie, seat belts, safety car seats, booster seats), and use of helmets while riding a bicycle or tricycle. We then assessed whether the child or parent had missed receiving guidance regarding at least 1 of the preventive counseling items in the past 2 years and during the child's lifetime. Lastly, we assessed whether the child had not received guidance regarding any of the 4 preventive counseling items (missing all 4) in the past 2 years and during the child's lifetime. Our analyses of the preventive counseling items excluded the 5,700 children in the MEPS-HC population that was younger than 2 years, because these questions were asked only of children aged 2 to 17 years.

\section{Independent Variables}

Among the MEPS-HC child sample ( $\mathrm{n}=56,302)$, we assessed the cross-sectional USC status of the child and parent(s) as the primary independent variable. We then created 6 mutually exclusive child-parent USC patterns (Table 1). These 6 groups were collapsed into 4 groups for univariate and multivariate analyses: (1) the child and 1 or both parent(s) have a USC (USC: yes child/yes parent $[\mathrm{s}]),(2)$ the child has a USC and 1 or both parents do not have a USC (USC: yes child/no parent), (3) the child does not have a USC and 1 or both parents have a USC (USC: no child/yes parent[s]), and (4) neither child nor parent has a USC (USC: no child/no parent).

Identification of the covariates that might influence 
children's access to health care was guided by the conceptual model designed by Aday and Andersen ${ }^{35,36}$ and by MEPS-HC variables shown to be associated with children's unmet health care needs. ${ }^{22}$ We used 2-tailed $\chi^{2}$ analyses to test univariate associations between these variables and each of the outcome variables. Ten independent variables were associated with at least 1 outcome $(P<.10)$ : household income, child's age, child's race/ethnicity, family composition, parental education, parental employment, region of residence, child's insurance status, parent's insurance status, and child's health status. Thus, all covariates were included in logistic regression models with the exception of parental employment, which was found to have significant collinearity with parental education and household income variables.

Specifically, the household income groups were based on the MEPS-HC-constructed variable that divides families into 5 income groups based on earnings as a percentage of the federal poverty level, and in 2007 the federal poverty level for a family of 4 was $\$ 20,650$. $^{37}$ We created 1 child race/ethnicity variable that was based on self-reported responses to standard options provided by MEPS interviewers. Family composition

\begin{tabular}{|c|c|c|c|}
\hline Family USC Patterns & $\begin{array}{l}\text { Unweighted } \\
\qquad \mathrm{N}^{\mathrm{a}}\end{array}$ & $\begin{array}{l}\text { Yearly Average } \\
\text { Weighted to } \\
\text { US Population } \\
\text { (in millions) }\end{array}$ & $\begin{array}{l}\text { Weighted \%b } \\
(95 \% \mathrm{CI})\end{array}$ \\
\hline \multicolumn{4}{|l|}{ Cross-sectional: 6 groups } \\
\hline \multicolumn{4}{|l|}{ Child yes USC } \\
\hline USC: yes parent(s) & 34,360 & 47.5 & $66.9(65.6-68.1)$ \\
\hline USC: yes 1 parent/no 1 parent & 6,894 & 8.3 & $11.7(11.0-12.3)$ \\
\hline USC: no parent(s) & 8,487 & 8.8 & $12.4(11.7-13.1)$ \\
\hline \multicolumn{4}{|l|}{ Child no USC } \\
\hline USC: yes parent(s) & 841 & 1.0 & $1.3(1.2-1.5)$ \\
\hline USC: yes 1 parent/no 1 parent & 788 & 0.8 & $1.2(1.0-1.3)$ \\
\hline USC: no parent(s) & 4,932 & 4.6 & $6.5(6.0-7.1)$ \\
\hline Total & 56,302 & 70.9 & 100.0 \\
\hline \multicolumn{4}{|l|}{ Cross-sectional: $\mathbf{4}$ groups } \\
\hline \multicolumn{4}{|l|}{ Child yes USC } \\
\hline USC: yes parent(s) & 41,254 & 55.7 & $78.6(77.5-79.6)$ \\
\hline USC: no parent(s) & 8,487 & 8.8 & $12.4(11.7-13.1)$ \\
\hline \multicolumn{4}{|l|}{ Child no USC } \\
\hline USC: yes parent(s) & 1,629 & 1.8 & $2.5(2.3-2.8)$ \\
\hline USC: no parent(s) & 4,932 & 4.6 & $6.5(6.0-7.1)$ \\
\hline Total & 56,302 & 70.9 & 100.0 \\
\hline
\end{tabular}

Source: 2002-2007 Medical Expenditure Panel Survey (MEPS), Household Component.

Note: Column percentages may not equal 100\% because of rounding (rounded to nearest 10 th).

a Unweighted counts represent total number of children, aged 0-17 years, from MEPS-respondent households with a positive person weight who could be linked to at least 1 parent within the household. Total counts do not include 2,049 children with no parent identified in the household. Total also excludes 437 children for whom USC status could not be ascertained for the child or the parent.

${ }^{\mathrm{b}}$ To derive the yearly population estimates, each child record from the MEPS was weighted according to person-level weights provided by the data collection agency. refers to whether the child could be linked to 1 parent or 2 parents residing in the same household (it does not account for biological relationship between parent and child or the marriage status between the 2 parents).

\section{Analyses}

We determined the prevalence of different childparent USC patterns. We then used 2 -tailed $\chi^{2}$ tests to ascertain univariate associations between covariates and each of the 4 child-parent USC subgroups, and univariate associations between each of the child-parent subgroups and unmet need and preventive counseling outcomes. Finally, we used a series of logistic regression models to assess the adjusted associations between the 4 child-parent USC subgroups and the outcomes. Models were first built for the outcome of having no doctor visits in the past 12 months using forward selection modeling techniques. Covariates selected in this model were used for all remaining outcomes to achieve consistency in our examination and to facilitate comparisons of results across models, with 1 exception-child's and parent's insurance status were excluded from the model that assessed insurance coverage gap as the outcome.

We reported measures of association as adjusted risk ratios (aRRs). ${ }^{38}$

We used SUDAAN 10.0.1 (Research Triangle Institute, Research Triangle Park, North Carolina) for all statistical analyses to account for the complex sampling design of the MEPS; $\alpha$ level was set at .05 for all multivariable analyses a priori. This study protocol was reviewed by the Oregon Health \& Science University Institutional Review Board, which deemed the study exempt because the MEPS-HC is publicly available.

\section{RESULTS}

Among the study population of children, $78.6 \%$ had a USC and at least 1 parent had a USC, $12.4 \%$ had a USC but neither parent had a USC, $2.5 \%$ had no USC but at least 1 parent had a USC, and $6.5 \%$ had no USC and no parent had a USC (Table 1).

All characteristics of children and their parents were significantly different across the 4 child-parent USC subgroups $(P<.05$; Table 2$)$. Most notably, the USC: yes child/ 
Table 2. Characteristics Associated With Different Cross-Sectional Family Usual Source of Care (USC) Patterns for US Children Aged 0 to 17 Years Living With at Least 1 Parent, 2002-2007

\begin{tabular}{|c|c|c|c|c|c|}
\hline \multirow[b]{2}{*}{ Characteristics } & \multirow[b]{2}{*}{ All Children } & \multicolumn{4}{|c|}{$\begin{array}{l}\text { Cross-Sectional Family USC Patterns: } 4 \text { Groups } \\
\text { (Does Not Account for Discordant Parent Status) }\end{array}$} \\
\hline & & $\begin{array}{l}\text { USC: Yes Childl } \\
\text { Yes Parent(s) }\end{array}$ & $\begin{array}{l}\text { USC: Yes Child } l \\
\text { No Parent }\end{array}$ & $\begin{array}{l}\text { USC: No ChildI } \\
\text { Yes Parent(s) }\end{array}$ & $\begin{array}{l}\text { USC: No Child } \\
\text { No Parent }\end{array}$ \\
\hline No. in sample ${ }^{a}$ & 56,302 & 41,254 & 8,487 & 1,629 & 4,932 \\
\hline \multicolumn{6}{|l|}{ Household income group, \% FPL ${ }^{\mathrm{b}, \mathrm{c}}$} \\
\hline$>400 \%$ & 28.8 & 31.9 & 18.5 & 18.9 & 15.0 \\
\hline $200 \%$ to $<400 \%$ & 33.1 & 34.4 & 26.7 & 34.0 & 29.4 \\
\hline $125 \%$ to $<200 \%$ & 15.8 & 14.6 & 19.4 & 22.1 & 21.5 \\
\hline $100 \%$ to $<125 \%$ & 5.4 & 4.7 & 7.7 & 7.3 & 8.3 \\
\hline$<100 \%$ & 17.0 & 14.5 & 27.7 & 17.8 & 25.7 \\
\hline \multicolumn{6}{|l|}{ Child's age, $y, \%^{b}$} \\
\hline $0-4$ & 27.4 & 26.7 & 39.5 & 12.9 & 17.7 \\
\hline $5-9$ & 27.4 & 27.5 & 28.7 & 18.7 & 26.0 \\
\hline $10-13$ & 22.4 & 22.6 & 18.8 & 23.7 & 25.3 \\
\hline $14-17$ & 22.9 & 23.2 & 12.9 & 44.7 & 31.0 \\
\hline \multicolumn{6}{|l|}{ Child's race/ethnicity, \% ${ }^{b, d}$} \\
\hline White, non-Hispanic & 58.9 & 63.1 & 46.1 & 44.9 & 38.1 \\
\hline Hispanic, any race & 19.7 & 16.5 & 29.4 & 25.6 & 37.0 \\
\hline Nonwhite, non-Hispanic & 21.4 & 20.4 & 24.5 & 29.5 & 25.0 \\
\hline \multicolumn{6}{|l|}{ Family composition, $\%^{\mathrm{b}, \mathrm{e}}$} \\
\hline 1 Parent in household & 26.2 & 23.5 & 38.6 & 24.7 & 36.8 \\
\hline 2 Parents in household & 73.8 & 76.6 & 61.4 & 75.3 & 63.2 \\
\hline \multicolumn{6}{|l|}{$\begin{array}{l}\text { At least } 1 \text { parent completed } \\
\text { high school, \% }\end{array}$} \\
\hline Yes & 85.9 & 88.6 & 77.9 & 78.2 & 71.9 \\
\hline No & 14.1 & 11.4 & 22.1 & 21.8 & 28.1 \\
\hline \multicolumn{6}{|l|}{ Geographic residence, $\%^{\mathrm{b}}$} \\
\hline Northeast & 17.5 & 19.8 & 10.3 & 8.2 & 6.9 \\
\hline West & 24.4 & 23.3 & 25.4 & 28.6 & 33.4 \\
\hline South & 35.9 & 33.0 & 47.6 & 45.8 & 45.4 \\
\hline Midwest & 22.2 & 23.9 & 16.7 & 17.4 & 14.3 \\
\hline \multicolumn{6}{|l|}{ Child's insurance status, \% ${ }^{\mathrm{b}}$} \\
\hline Full year insured & 81.8 & 85.7 & 76.9 & 64.1 & 50.9 \\
\hline Insurance gap & 11.4 & 9.2 & 16.4 & 18.5 & 24.6 \\
\hline Full year uninsured & 6.8 & 5.1 & 6.6 & 17.4 & 24.6 \\
\hline \multicolumn{6}{|l|}{ Parent's insurance status, $\%^{\mathrm{b}}$} \\
\hline Insured all year (at least 1 parent) & 75.6 & 82.1 & 51.6 & 68.8 & 45.4 \\
\hline Not insured all year & 24.4 & 17.9 & 48.5 & 31.2 & 54.6 \\
\hline \multicolumn{6}{|l|}{ Child health status, $\%^{\mathrm{b}}$} \\
\hline Excellent/very good & 82.3 & 83.3 & 78.5 & 82.5 & 78.2 \\
\hline Good/fair/poor & 17.7 & 16.7 & 21.5 & 17.5 & 21.8 \\
\hline
\end{tabular}

$\mathrm{FPL}=$ federal poverty level; MEPS-HC = Medical Expenditure Panel Survey-Household Component.

Source: 2002-2007 MEPS-HC.

Note: Column percentages are weighted and may not equal $100 \%$ because of rounding (rounded to nearest 10 th). $P<.05$ in $\chi^{2}$ test comparisons of overall differences between subgroups of each covariate and insurance patterns.

a Unweighted counts represent total number of children, aged 0-17 years, from MEPS-HC respondent households with a positive person weight who could be linked to at least 1 parent within the household. Total counts do not include 2,049 children with no parent identified in the household; cross-sectional total also excludes 437 children for whom self or parental USC status could not be ascertained.

${ }^{b}$ To derive yearly population estimates, each child record from MEPS-HC was weighted according to person-level weights provided by the data collection agency. 'Household income groups were based on MEPS-HC-constructed variable that divides families into 5 income groups based on earnings as a percentage of federal poverty level (FPL), and in 2007, the federal poverty level for a family of 4 was $\$ 20,650$. . $^{37}$

${ }^{d}$ Child's race/ethnicity based on responses to standard options provided by MEPS-HC interviewers. We created 1 child race/ethnicity variable by combining a race variable (which included white only, black only, American Indian/Alaskan Native only, Asian only, native Hawaiian/Pacific Islander only, and multiple races) and an ethnicity variable (which included Hispanic, or not Hispanic).

e Family composition refers to whether the child could be linked to 1 parent $(n=17,612)$ or 2 parents $(n=38,690)$ residing in the same household (does not account for biological relationship between parent and child or the marriage status between the 2 parents). 
yes parent(s) subgroup had the highest percentage of families earning more than $400 \%$ of the federal poverty level. A higher percentage of the USC: no child/ no parent subgroup was Hispanic compared with all children. Children with unstable insurance coverage were also more likely to be in the USC: no child/no parent subgroup. Other characteristics disproportionately associated with the USC: no child/no parent subgroup included older age of the child, single-parent households, having parent(s) who did not complete high school, and having uninsured parents.

The percentage of children with unmet needs increased sharply as child-parent USC patterns deviated from the concordant pattern of both child and parent(s) having a USC (Table 3 ). In all cases, the
USC: yes child/yes parent(s) subgroup had the lowest rates of unmet health care needs; children in the USC: no child/no parent subgroup had the highest rates. In univariate analyses, the USC: yes child/no parent subgroup had higher percentages of unmet health care needs than did the USC: yes child/yes parent(s) subgroup for all outcomes examined. The same pattern held true for the 4 preventive counseling items.

In multivariate analyses, the higher rates of unmet needs among children in the USC: yes child/no parent subgroup when compared with the USC: yes child/ yes parent(s) subgroup achieved statistical significance in almost all cases. As shown in Table 4, the USC: yes child/no parent subgroup had a higher likelihood of an insurance coverage gap $(\mathrm{aRR}=1.33 ; 95 \%$ confi-

\section{Table 3. Univariate Associations Between Cross-Sectional Family Usual Source of Care (USC) Patterns} and Children's Receipt of Health Care Services, 2002-2007

\begin{tabular}{|c|c|c|c|c|c|}
\hline Outcomes & $\begin{array}{l}\text { All Children } \\
\text { Weighted \% } \\
(95 \% \mathrm{CI})^{\mathrm{a}}\end{array}$ & $\begin{array}{l}\text { USC: Yes Childl } \\
\text { Yes Parent(s) } \\
\text { Weighted \% } \\
(95 \% \mathrm{Cl})^{\mathrm{a}}\end{array}$ & $\begin{array}{c}\text { USC: Yes Child I } \\
\text { No Parent } \\
\text { Weighted \% } \\
(95 \% \mathrm{Cl})^{\mathrm{a}}\end{array}$ & $\begin{array}{l}\text { USC: No Childl } \\
\text { Yes Parent(s) } \\
\text { Weighted \% } \\
(95 \% \mathrm{Cl})^{\mathrm{a}}\end{array}$ & $\begin{array}{l}\text { USC: No Child } I \\
\text { No Parent } \\
\text { Weighted \% } \\
(95 \% \mathrm{Cl})^{\mathrm{a}}\end{array}$ \\
\hline \multicolumn{6}{|l|}{ Cross-sectional: 4 groups } \\
\hline No. in sample ${ }^{b}$ & 56,302 & 41,254 & 8,487 & 1,629 & 4,932 \\
\hline Child had insurance coverage gap & $18.2(17.4-19.1)$ & $14.3(13.6-15.1)$ & $23.1(21.2-25.1)$ & $35.9(32.0-40.0)$ & $49.1(46.3-52.0)$ \\
\hline No doctor visits in past year & $23.0(22.2-23.9)$ & $19.1(18.3-19.9)$ & $22.6(20.9-24.4)$ & $54.6(51.0-58.1)$ & $58.9(56.4-61.4)$ \\
\hline No dental visits at least yearly & $27.6(26.7-28.4)$ & $24.7(23.9-25.6)$ & $37.3(35.2-39.3)$ & $35.6(31.9-39.5)$ & $40.1(37.5-42.7)$ \\
\hline $\begin{array}{l}\text { Unmet medical or prescription } \\
\text { needc }\end{array}$ & $1.4(1.2-1.6)$ & $1.1(0.9-1.2)$ & $2.1(1.5-3.1)$ & $2.5(1.6-4.0)$ & $3.6(2.8-4.6)$ \\
\hline Delayed care ${ }^{d}$ & $31.0(30.0-32.0)$ & $30.2(29.2-31.2)$ & $33.1(30.9-35.5)$ & $33.8(29.0-38.9)$ & $40.6(37.1-44.1)$ \\
\hline Problems getting care ${ }^{e}$ & $15.6(14.8-16.5)$ & $14.5(13.7-15.4)$ & $17.8(15.5-20.4)$ & $28.1(22.2-34.8)$ & $31.4(27.0-36.3)$ \\
\hline \multicolumn{6}{|l|}{ Cross-sectional: $\mathbf{4}$ groups } \\
\hline No. in sample ${ }^{b}$ & 50,602 & 37,175 & 7,201 & 1,566 & 4,660 \\
\hline \multicolumn{6}{|l|}{ Past 2 years } \\
\hline $\begin{array}{l}\text { Did not receive all } 4 \text { preventive } \\
\text { counseling items (missing }>1)^{f, g}\end{array}$ & $83.2(82.4-84.0)$ & $82.0(81.0-83.0)$ & $85.4(83.8-86.8)$ & $90.2(87.2-92.5)$ & $90.8(89.0-92.3)$ \\
\hline $\begin{array}{l}\text { Did not receive any of } 4 \text { preventive } \\
\text { counseling items (missing all } 4)^{f}\end{array}$ & $39.8(38.8-40.9)$ & $37.2(36.1-38.3)$ & $40.0(37.6-42.4)$ & $62.5(58.6-66.3)$ & $61.6(59.2-63.9)$ \\
\hline \multicolumn{6}{|l|}{ Lifetime } \\
\hline $\begin{array}{l}\text { Did not receive all } 4 \text { preventive } \\
\text { counseling items (missing }>1)^{\text {th }}\end{array}$ & $80.0(78.9-80.9)$ & $78.6(77.4-79.6)$ & $82.9(81.3-84.5)$ & $87.1(83.8-89.8)$ & $87.9(85.9-89.6)$ \\
\hline $\begin{array}{l}\text { Did not receive any of } 4 \text { preventive } \\
\text { counseling items (missing all } 4)^{f}\end{array}$ & $33.4(32.3-34.5)$ & $31.0(29.9-32.1)$ & $33.8(31.6-36.1)$ & $53.5(49.5-57.5)$ & $52.8(50.2-55.5)$ \\
\hline
\end{tabular}

Source: 2002-2007 Medical Expenditure Panel Survey-Household Component (MEPS-HC).

Note: $P<.05$ in $\chi^{2}$ test comparisons of overall differences between insurance pattern subgroups and all outcome measures.

${ }^{a}$ To derive the yearly population estimates, each child record from the MEPS-HC was weighted according to person-level weights provided by the data collection agency. b Unweighted counts represent total number of children, aged 0-17 years, from MEPS-HC-respondent households with a positive person weight who could be linked to at least 1 parent within the household. Total counts do not include 2,049 children with no parent identified in the household. Total also excludes 437 children for whom USC status could not be ascertained for the child and/or parent.

' Unmet medical or prescription need was defined as being unable to get medical care and/or prescription medications when needed within the past year.

${ }^{d}$ Delayed care was defined as parental report that child did not always get care as soon as was wanted for an illness, injury, or condition that needed care right away; or did not always get an appointment for routine health care as soon as was wanted.

e Problems getting care was defined as parent reporting a big or small problem that child did not receive needed medical or prescription treatment; a big or small problem to get the child care, tests, or treatment a parent or doctor believed necessary; or a big or small problem to see a specialist the child needed to see.

${ }^{f}$ The preventive counseling services include MEPS-HC items that asked parents whether a doctor or health care clinician had advised their child about the importance of (1) healthy eating, (2) routine exercise, (3) use of car safety seats/booster seats/seat belts, and (4) use of a helmet while riding a tricycle/bicycle.

${ }^{g}$ In the past 2 years among all children, $39.8 \%$ were missing all 4 preventive counseling items; $16.7 \%$ were missing 3 items; $15.3 \%$ were missing 2 items; $11.4 \%$ were missing 1 item ( $16.8 \%$ had received all 4 items).

${ }^{\mathrm{h}}$ Over a lifetime among all children, $33.4 \%$ were missing all 4 preventive counseling items; $16.9 \%$ were missing 3 items; $16.5 \%$ were missing 2 items; $13.2 \%$ were missing 1 item $(20.1 \%$ had received all 4 items). 
dence interval $[\mathrm{CI}], 1.21-1.47)$, having no doctor visits in the past 12 months $(\mathrm{aRR}=1.11 ; 95 \% \mathrm{CI}, 1.02-1.21)$, less than yearly dental visits $(\mathrm{aRR}=1.12 ; 95 \% \mathrm{CI}$, 1.06-1.18), and unmet medical or prescription needs
$(\mathrm{aRR}=1.70 ; 95 \% \mathrm{CI}, 1.09-2.65)$, when compared with the USC: yes child/yes parent(s) subgroup.

In Table 5, the USC: yes child/no parent subgroup was also at higher risk of having not received preven-

\section{Table 4. Multivariate Associations Between Child and Family Characteristics and Children's Access to Health Care (2002-2007)}

\begin{tabular}{|c|c|c|c|c|c|c|}
\hline $\begin{array}{l}\text { Demographic } \\
\text { and Other } \\
\text { Characteristics } \\
\end{array}$ & $\begin{array}{l}\text { Child Health } \\
\text { Insurance } \\
\text { Coverage Gap } \\
\text { aRR }(95 \% \mathrm{Cl}) \\
\end{array}$ & $\begin{array}{l}\text { No Doctor } \\
\text { Visits in } \\
\text { Past } 12 \mathrm{Mo}^{\mathrm{a}} \\
\text { aRR }(95 \% \mathrm{Cl})\end{array}$ & $\begin{array}{c}\text { Child } \\
\text { Dentist } \\
\text { Visits }<1 / y^{b} \\
\text { aRR }(95 \% \text { Cl) }\end{array}$ & $\begin{array}{c}\text { Unmet Medical } \\
\text { or Prescription } \\
\text { Needc } \\
\text { aRR }(95 \% \mathrm{Cl})\end{array}$ & $\begin{array}{c}\text { Delayed } \\
\text { Care }^{\mathrm{d}} \\
\text { aRR }(95 \% \mathrm{Cl})\end{array}$ & $\begin{array}{c}\text { Problems } \\
\text { Getting Care } \\
\text { aRR (95\% Cl) }\end{array}$ \\
\hline \multicolumn{7}{|l|}{ Family USC patterns } \\
\hline $\begin{array}{l}\text { USC: yes child/ } \\
\text { yes parent(s) }\end{array}$ & 1.00 & 1.00 & 1.00 & 1.00 & 1.00 & 1.00 \\
\hline $\begin{array}{l}\text { USC: yes child/ } \\
\text { no parent }\end{array}$ & $1.33(1.21-1.47)^{g}$ & $1.11(1.02-1.21)^{g}$ & $1.12(1.06-1.18)^{g}$ & $1.70(1.09-2.65)^{\mathrm{g}}$ & $1.00(0.93-1.08)$ & $1.06(0.91-1.23)$ \\
\hline $\begin{array}{l}\text { USC: no child/ } \\
\text { yes parent(s) }\end{array}$ & $2.15(1.92-2.41)^{\mathrm{g}}$ & $2.19(2.02-2.38)^{9}$ & $1.40(1.28-1.52)^{9}$ & $1.63(0.98-2.69)$ & $1.05(0.91-1.21)$ & $1.65(1.32-2.07)^{\mathrm{g}}$ \\
\hline $\begin{array}{l}\text { USC: no child/ } \\
\text { no parent }\end{array}$ & $2.82(2.60-3.04)^{9}$ & $2.35(2.22-2.50)^{9}$ & $1.38(1.30-1.47)^{9}$ & $2.00(1.51-2.66)^{9}$ & $1.19(1.08-1.32)^{g}$ & $1.70(1.45-2.00)^{9}$ \\
\hline \multicolumn{7}{|l|}{$\begin{array}{l}\text { Household income } \\
\text { group, \% FPL }\end{array}$} \\
\hline$>400 \%^{4}$ & 1.00 & 1.00 & 1.00 & 1.00 & 1.00 & 1.00 \\
\hline $200 \%-<400 \%$ & $1.57(1.40-1.76)^{9}$ & $1.47(1.37-1.58)^{9}$ & $1.26(1.19-1.33)^{g}$ & $1.91(1.30-2.80)^{9}$ & $1.03(0.97-1.09)$ & $1.25(1.11-1.41)^{\mathrm{g}}$ \\
\hline $125 \%$ to $<200 \%$ & $2.09(1.86-2.35)^{g}$ & $1.63(1.49-1.77)^{9}$ & $1.44(1.34-1.55)^{9}$ & $2.13(1.51-3.01)^{g}$ & $1.03(0.95-1.11)$ & $1.31(1.15-1.50)^{\mathrm{g}}$ \\
\hline $100 \%$ to $<125 \%$ & $2.05(1.76-2.39)^{9}$ & $1.66(1.51-1.84)^{9}$ & $1.42(1.30-1.56)^{9}$ & $2.32(1.42-3.81)^{9}$ & $1.07(0.96-1.18)$ & $1.36(1.12-1.64)^{\mathrm{g}}$ \\
\hline$<100 \%$ & $1.67(1.47-1.91)^{g}$ & $1.60(1.46-1.75)^{9}$ & $1.40(1.30-1.51)^{\mathrm{g}}$ & $2.19(1.50-3.20)^{g}$ & $1.09(1.00-1.19)$ & $1.50(1.29-1.74)^{\mathrm{g}}$ \\
\hline \multicolumn{7}{|l|}{ Child's age, y } \\
\hline $0-4^{f}$ & 1.00 & 1.00 & 1.00 & 1.00 & 1.00 & 1.00 \\
\hline $5-9$ & $0.99(0.92-1.06)$ & $2.12(1.98-2.29)^{9}$ & $0.22(0.21-0.24)^{9}$ & $1.38(1.07-1.78)^{g}$ & $1.03(0.98-1.09)$ & $1.05(0.96-1.16)$ \\
\hline $10-13$ & $1.05(0.97-1.13)$ & $2.39(2.20-2.58)^{9}$ & $0.20(0.19-0.22)^{9}$ & $1.26(0.90-1.76)$ & $1.04(0.97-1.11)$ & $1.06(0.94-1.19)$ \\
\hline $14-17$ & $1.06(0.98-1.15)$ & $2.50(2.32-2.69)^{9}$ & $0.30(0.28-0.32)^{9}$ & $1.48(1.06-2.07)^{\mathrm{g}}$ & $1.09(1.02-1.16)^{9}$ & $1.16(1.04-1.30)^{\mathrm{g}}$ \\
\hline \multicolumn{7}{|l|}{ Child's race/ethnicity } \\
\hline $\begin{array}{l}\text { White, non- } \\
\text { Hispanic }^{f}\end{array}$ & 1.00 & 1.00 & 1.00 & 1.00 & 1.00 & 1.00 \\
\hline Hispanic, any race & $1.34(1.23-1.47)^{9}$ & $1.17(1.09-1.26)^{9}$ & $0.99(0.93-1.05)$ & $0.56(0.40-0.78)^{g}$ & $1.09(1.01-1.17)^{\mathrm{g}}$ & $1.07(0.96-1.18)$ \\
\hline $\begin{array}{l}\text { Nonwhite, } \\
\text { non-Hispanic }\end{array}$ & $0.86(0.77-0.95)^{9}$ & $1.24(1.16-1.32)^{9}$ & $1.00(0.95-1.05)$ & $0.74(0.57-0.97)^{9}$ & $1.04(0.98-1.11)$ & $1.06(0.94-1.19)$ \\
\hline \multicolumn{7}{|l|}{ Family composition } \\
\hline $\begin{array}{l}2 \text { Parents in } \\
\text { household }^{f}\end{array}$ & 1.00 & 1.00 & 1.00 & 1.00 & 1.00 & 1.00 \\
\hline $\begin{array}{l}1 \text { Parent in } \\
\text { household }\end{array}$ & $1.09(1.01-1.18)^{\mathrm{g}}$ & $0.92(0.88-0.97)^{9}$ & $1.04(0.99-1.09)$ & $1.19(0.92-1.54)$ & $1.07(1.02-1.13)^{g}$ & $1.03(0.93-1.14)$ \\
\hline \multicolumn{7}{|l|}{$\begin{array}{l}\text { At least } 1 \text { parent } \\
\text { completed high } \\
\text { school }\end{array}$} \\
\hline Yes $^{f}$ & 1.00 & 1.00 & 1.00 & 1.00 & 1.00 & 1.00 \\
\hline No & $1.14(1.04-1.25)^{9}$ & $1.22(1.14-1.30)^{9}$ & $1.19(1.12-1.26)^{g}$ & $0.82(0.62-1.07)$ & $1.06(0.99-1.14)$ & $0.87(0.77-1.00)$ \\
\hline \multicolumn{7}{|l|}{$\begin{array}{l}\text { Geographic } \\
\text { residence }\end{array}$} \\
\hline $\begin{array}{l}\text { Northeast (refer- } \\
\text { ence group) }\end{array}$ & 1.00 & 1.00 & 1.00 & 1.00 & 1.00 & 1.00 \\
\hline West & $1.26(1.10-1.44)^{9}$ & $1.68(1.48-1.90)^{9}$ & $1.14(1.04-1.24)^{9}$ & $0.99(0.64-1.52)$ & $1.31(1.19-1.44)^{g}$ & $1.10(0.94-1.28)$ \\
\hline South & $1.38(1.21-1.57)^{g}$ & $1.39(1.23-1.57)^{9}$ & $1.14(1.05-1.23)^{\mathrm{g}}$ & $0.98(0.70-1.36)$ & $1.12(1.02-1.23)^{9}$ & $0.93(0.81-1.06)$ \\
\hline Midwest & $1.13(0.99-1.29)$ & $1.42(1.25-1.61)^{\mathrm{g}}$ & $1.12(1.02-1.22)^{g}$ & $1.00(0.70-1.43)$ & $1.19(1.08-1.31)^{g}$ & $0.90(0.77-1.05)$ \\
\hline \multicolumn{7}{|l|}{$\begin{array}{l}\text { Parent's insurance } \\
\text { status }\end{array}$} \\
\hline $\begin{array}{l}\text { Insured all year } \\
\text { (at least } 1)^{\dagger}\end{array}$ & $\mathrm{N} / \mathrm{A}$ & 1.00 & 1.00 & 1.00 & 1.00 & 1.00 \\
\hline \multirow[t]{2}{*}{$\begin{array}{l}\text { Not insured all } \\
\text { year }\end{array}$} & $\mathrm{N} / \mathrm{A}$ & $1.03(0.97-1.09)$ & $0.97(0.91-1.03)$ & $1.14(0.89-1.46)$ & $1.08(1.01-1.15)$ & $1.11(0.99-1.25)$ \\
\hline & & & & & & Table 4 continues \\
\hline
\end{tabular}


Table 4. Multivariate Associations Between Child and Family Characteristics and Children's Access to Health Care (2002-2007) continued

\begin{tabular}{|c|c|c|c|c|c|c|}
\hline $\begin{array}{l}\text { Demographic } \\
\text { and Other } \\
\text { Characteristics }\end{array}$ & $\begin{array}{l}\text { Child Health } \\
\text { Insurance } \\
\text { Coverage Gap } \\
\text { aRR }(95 \% \mathrm{Cl}) \\
\end{array}$ & $\begin{array}{l}\text { No Doctor } \\
\text { Visits in } \\
\text { Past } 12 \mathrm{Mo}^{\mathrm{a}} \\
\text { aRR }(95 \% \mathrm{Cl})\end{array}$ & $\begin{array}{c}\text { Child } \\
\text { Dentist } \\
\text { Visits }<1 / y^{b} \\
\text { aRR }(95 \% \mathrm{Cl})\end{array}$ & $\begin{array}{c}\text { Unmet Medical } \\
\text { or Prescription } \\
\text { Needc } \\
\text { aRR }(95 \% \mathrm{Cl})\end{array}$ & $\begin{array}{c}\text { Delayed } \\
\text { Care }^{\mathrm{d}} \\
\text { aRR }(95 \% \mathrm{Cl})\end{array}$ & $\begin{array}{l}\text { Problems } \\
\text { Getting Care } \\
\text { aRR }(95 \% \mathrm{Cl})\end{array}$ \\
\hline \multicolumn{7}{|l|}{$\begin{array}{l}\text { Child's insurance } \\
\text { status }\end{array}$} \\
\hline Insured all year & $N / A$ & 1.00 & 1.00 & 1.00 & 1.00 & 1.00 \\
\hline $\begin{array}{c}\text { Partial year } \\
\text { insurance }\end{array}$ & N/A & $1.19(1.11-1.27)^{g}$ & $1.37(1.29-1.45)^{\mathrm{g}}$ & $2.65(2.11-3.33)^{9}$ & $1.06(0.99-1.14)$ & $1.28(1.13-1.46)^{9}$ \\
\hline Full year uninsured & $\mathrm{N} / \mathrm{A}$ & $1.47(1.37-1.59)^{9}$ & $1.80(1.69-1.91)^{g}$ & $3.47(2.69-4.46)^{9}$ & $1.00(0.90-1.11)$ & $1.49(1.29-1.73)^{g}$ \\
\hline \multicolumn{7}{|l|}{ Child health status } \\
\hline $\begin{array}{l}\text { Excellent/ } \\
\text { very good }\end{array}$ & 1.00 & 1.00 & 1.00 & 1.00 & 1.00 & 1.00 \\
\hline Good/fair/poor & $1.03(0.97-1.09)$ & $0.74(0.70-0.79)^{\mathrm{g}}$ & $1.09(1.05-1.14)^{\mathrm{g}}$ & $2.35(1.87-2.96)^{9}$ & $1.26(1.19-1.32)^{\mathrm{g}}$ & $1.68(1.54-1.83)^{9}$ \\
\hline
\end{tabular}

Source: 2002-2007 Medical Expenditure Panel Survey-Household Component.

$\mathrm{aRR}=$ adjusted risk ratio; $\mathrm{FPL}=$ federal poverty level; $\mathrm{USC}=$ usual source of care.

a Yearly doctor visits were chosen as an unmet need variable because the American Academy of Pediatrics recommends yearly preventive pediatric health care visits up to age 21 years. ${ }^{32}$

${ }^{\mathrm{b}}$ American Academy of Pediatric Dentistry recommends yearly dental visits begin at time of first tooth and no later than 12 months of age. ${ }^{33}$

' Unmet medical or prescription need was defined as being unable to get medical care and/or prescription medications when needed within the past year.

¿ Delayed care was defined as parental report that child did not always get care as soon as was wanted for an illness, injury, or condition that needed care right away; or did not always get an appointment for routine health care as soon as was wanted.

e Problems getting care was defined as parent reporting a big or small problem that the child did not receive needed medical or prescription treatment; a big or small problem to get the child care, tests, or treatment a parent or doctor believed necessary; or a big or small problem to see a specialist the child needed to see. ${ }^{f}$ Reference group.

${ }^{g} P<.05$.

tive counseling when compared with the USC: yes child/yes parent(s) subgroup in 3 of the 4 models. Although group differences in Tables 4 and 5 were not statistically significant for all outcomes, the observed trends suggest increased vulnerability as USC patterns deviated from both child and parent(s) having a USC.

\section{DISCUSSION}

In aggregated cross-sectional MEPS-HC data from 2002 to 2007, 9\% of children (aged 17 years and younger and living with at least 1 parent) in the United States had no USC, and more than $18 \%$ of children had no parent in the household with a USC. In this study, the largest differences in children's receipt of necessary health care were between children with a USC and those without a USC, which confirms previous evidence about the importance of a USC for children. ${ }^{13-9}$ This study revealed the new finding that parental USC status is also associated with children's receipt of health care services, regardless of child's USC status. Among children with a USC, those whose parent(s) lacked a USC had a higher likelihood of experiencing unmet health care needs.

Our previous work has shown that insured children with uninsured parents have higher odds of unmet health care and preventive counseling needs when compared with insured children whose parents are insured. ${ }^{39}$ We have also shown that adults and children are both more likely to have unmet health care needs when they lack a USC, even if they have health insurance. ${ }^{40,41}$ In this study, we report on the importance of a USC for both parent and child, even while controlling for parental and child health insurance.

\section{Policy Implications}

The results of this study_-showing that a child's receipt of recommended health care services is associated not only with their own access to a USC but also their parents' access to a USC — highlight the need to broaden the focus beyond expansions in the child health care workforce and quality improvements in child health care to consider the role that parental access to a USC plays in ensuring access to health care services for children. There is a clear need to advocate for policy changes that improve access to a USC for all family members. The Patient Protection and Affordable Care Act (PPACA) has a provision to increase the number of primary care clinicians, ${ }^{42}$ which is a step in the right direction. Specifically, the PPACA will provide resources for increasing training through additional primary care residency programs and numbers of nurse practitioners, as well as training more physician assistants to work in primary care settings. Scholarship and loan repayment programs will also be implemented for primary care clinicians who choose to 
Table 5. Multivariate Associations Between Child and Family Characteristics and Children's Receipt of Preventive Counseling (2002-2007)

\begin{tabular}{|c|c|c|c|c|}
\hline \multirow[b]{2}{*}{$\begin{array}{l}\text { Demographic and } \\
\text { Other Characteristics }\end{array}$} & \multicolumn{2}{|c|}{ Past 2 Years } & \multicolumn{2}{|c|}{ Lifetime } \\
\hline & $\begin{array}{c}\text { Did Not Receive All } 4 \\
\text { Preventive Counseling } \\
\text { Items (Missing } \geq 1)^{\mathrm{a}} \\
\text { aRR }(95 \% \mathrm{CI})\end{array}$ & $\begin{array}{l}\text { Did Not Receive } \\
\text { Any of } 4 \text { Preventive } \\
\text { Counseling Items } \\
\text { aRR }(95 \% \mathrm{CI})\end{array}$ & $\begin{array}{l}\text { Did Not Receive All } 4 \\
\text { Preventive Counseling } \\
\text { Items (Missing } \geq 1)^{\mathrm{a}} \\
\text { aRR }(95 \% \mathrm{Cl})\end{array}$ & $\begin{array}{l}\text { Did Not Receive } \\
\text { Any of } 4 \text { Preventive } \\
\text { Counseling Items } \\
\text { aRR }(95 \% \mathrm{CI})\end{array}$ \\
\hline \multicolumn{5}{|l|}{ Family USC patterns } \\
\hline USC: yes child/yes parent(s) & 1.00 & 1.00 & 1.00 & 1.00 \\
\hline USC: yes child/no parent & $1.03(1.01-1.05)^{c}$ & $1.07(1.01-1.14)^{c}$ & $1.04(1.01-1.06)^{c}$ & $1.06(0.99-1.13)$ \\
\hline USC: no child/yes parent(s) & $1.07(1.03-1.11)^{c}$ & $1.49(1.38-1.61)^{c}$ & $1.08(1.04-1.12)^{c}$ & $1.52(1.39-1.66)^{c}$ \\
\hline USC: no child/no parent & $1.08(1.05-1.11)^{c}$ & $1.47(1.40-1.55)^{c}$ & $1.09(1.06-1.12)^{c}$ & $1.49(1.39-1.60)^{c}$ \\
\hline \multicolumn{5}{|l|}{ Household income group, \% FPL } \\
\hline$>400 \%{ }^{b}$ & 1.00 & 1.00 & 1.00 & 1.00 \\
\hline $200 \%-<400 \%$ & $1.05(1.02-1.07)^{c}$ & $1.23(1.17-1.30)^{c}$ & $1.05(1.03-1.08)^{c}$ & $1.27(1.19-1.35)^{c}$ \\
\hline $125 \%$ to $<200 \%$ & $1.04(1.01-1.07)^{c}$ & $1.24(1.16-1.32)^{c}$ & $1.06(1.03-1.09)^{c}$ & $1.31(1.21-1.41)^{c}$ \\
\hline $100 \%$ to $<125 \%$ & $1.03(1.00-1.07)$ & $1.22(1.12-1.33)^{c}$ & $1.04(1.01-1.08)^{c}$ & $1.27(1.15-1.40)^{c}$ \\
\hline$<100 \%$ & $1.02(0.99-1.05)$ & $1.17(1.10-1.25)^{c}$ & $1.03(1.00-1.06)$ & $1.23(1.14-1.33)^{c}$ \\
\hline \multicolumn{5}{|l|}{ Child's age, y } \\
\hline $2-4^{b}$ & 1.00 & 1.00 & 1.00 & 1.00 \\
\hline $5-9$ & $0.97(0.96-0.99)^{c}$ & $1.35(1.29-1.42)^{c}$ & $0.95(0.93-.096)^{c}$ & $1.27(1.21-1.35)^{c}$ \\
\hline $10-13$ & $1.00(0.98-1.02)$ & $1.52(1.44-1.61)^{c}$ & $0.96(0.94-0.98)^{c}$ & $1.43(1.34-1.51)^{c}$ \\
\hline $14-17$ & $1.06(1.04-1.07)^{c}$ & $1.70(1.61-1.81)^{c}$ & $1.01(0.99-1.03)$ & $1.55(1.44-1.65)^{c}$ \\
\hline \multicolumn{5}{|l|}{ Child's race/ethnicity } \\
\hline White, non-Hispanic ${ }^{b}$ & 1.00 & 1.00 & 1.00 & 1.00 \\
\hline Hispanic, any race & $0.95(0.93-0.97)^{c}$ & $0.88(0.83-0.93)^{c}$ & $0.95(0.93-0.98)^{c}$ & $0.94(0.89-1.00)$ \\
\hline Nonwhite, non-Hispanic & $0.99(0.98-1.01)$ & $0.96(0.91-1.02)$ & $1.01(0.99-1.03)$ & $1.02(0.96-1.09)$ \\
\hline \multicolumn{5}{|l|}{ Family composition } \\
\hline 2 Parents in household ${ }^{\mathrm{b}}$ & 1.00 & 1.00 & 1.00 & 1.00 \\
\hline 1 Parent in household & $1.02(1.01-1.04)^{c}$ & $0.99(0.95-1.03)$ & $1.03(1.01-1.04)^{c}$ & $0.98(0.94-1.04)$ \\
\hline \multicolumn{5}{|l|}{$\begin{array}{l}\text { At least } 1 \text { parent completed } \\
\text { high school }\end{array}$} \\
\hline Yes $^{b}$ & 1.00 & 1.00 & 1.00 & 1.00 \\
\hline No & $1.00(0.98-1.02)$ & $1.09(1.04-1.15)^{c}$ & $1.01(0.99-1.04)$ & $1.12(1.06-1.19)^{c}$ \\
\hline \multicolumn{5}{|l|}{ Geographic residence } \\
\hline Northeast ${ }^{b}$ & 1.00 & 1.00 & 1.00 & 1.00 \\
\hline West & $1.10(1.06-1.14)^{c}$ & $1.40(1.26-1.56)^{c}$ & $1.08(1.03-1.13)^{c}$ & $1.29(1.14-1.46)^{c}$ \\
\hline South & $1.12(1.08-1.16)^{c}$ & $1.37(1.24-1.52)^{c}$ & $1.12(1.08-1.16)^{c}$ & $1.31(1.16-1.47)^{c}$ \\
\hline Midwest & $1.11(1.07-1.15)^{c}$ & $1.36(1.22-1.51)^{c}$ & $1.11(1.07-1.16)^{c}$ & $1.29(1.14-1.46)^{c}$ \\
\hline \multicolumn{5}{|l|}{ Parent's insurance status } \\
\hline $\begin{array}{l}\text { Insured all year (at least } \\
1 \text { parent) }\end{array}$ & 1.00 & 1.00 & 1.00 & 1.00 \\
\hline Not insured all year & $1.01(0.99-1.03)$ & $1.02(0.97-1.07)$ & $1.01(0.99-1.03)$ & $1.03(0.98-1.09)$ \\
\hline \multicolumn{5}{|l|}{ Child's insurance status } \\
\hline Insured all yearb & 1.00 & 1.00 & 1.00 & 1.00 \\
\hline Partial year insurance & $1.04(1.01-1.06)^{c}$ & $1.11(1.06-1.18)^{c}$ & $1.04(1.01-1.06)^{c}$ & $1.10(1.03-1.17)^{c}$ \\
\hline Full year uninsured & $1.05(1.02-1.08)^{c}$ & $1.23(1.15-1.31)^{c}$ & $1.05(1.02-1.08)^{c}$ & $1.20(1.11-1.29)^{c}$ \\
\hline \multicolumn{5}{|l|}{ Child health status } \\
\hline Excellent/very good ${ }^{\mathrm{b}}$ & 1.00 & 1.00 & 1.00 & 1.00 \\
\hline Good/fair/poor & $1.01(0.99-1.03)$ & $0.90(0.86-0.94)^{c}$ & $1.02(1.00-1.04)$ & $0.92(0.88-0.96)^{c}$ \\
\hline
\end{tabular}

aRR = adjusted risk ratio; $F P L=$ federal poverty level; $\mathrm{USC}=$ usual source of care.

Source: 2002-2007 Medical Expenditure Panel Survey-Household Component (MEPS-HC).

a The preventive counseling services include MEPS-HC items that asked parents whether a doctor or health care clinician had advised their child about the importance of (1) healthy eating, (2) routine exercise, (3) use of care safety seats/booster seats/seat belts, and (4) use of a helmet while riding a tricycle/bicycle.

${ }^{b}$ Reference group.

c $P<.05$. 
work in underserved areas. ${ }^{43}$ Another possible policy change that could affect USC continuity is ensuring that a change in health insurance plans does not mean a change in one's primary care clinician or site.

Furthermore, as more children and parents have discordant insurance coverage patterns (eg, child covered by public insurance, parents covered by employerbased private insurance) ${ }_{1}^{44}$ the need for both child and parents to have a USC will become even more important. Further studies are needed to determine which aspects of care are most likely to improve when everyone in the family has a USC, and whether childparent USC concordance might mitigate the impact of fragmented family insurance coverage.

\section{Practice Implications}

In addition to advocating for the important policy changes outlined above, clinicians can develop practice-based interventions to assist parents in finding a stable USC for their children and for themselves. Some clinicians, such as family physicians and general practitioners, are in the unique position of providing primary care to all members of the same family. The patientcentered medical home holds promise as a model that can focus on coordinating care for entire families. ${ }^{10}$

Even clinicians who see only children or only adults could perform similar interventions if they are part of larger centers that can provide coordinated care for all family members. Interventions could also involve methods to track USC status for all family members. With widespread and expanding implementation of electronic health record systems, such processes and potential interventions could be computerized, and family records could be linked across USC sites.

\section{Limitations}

Although the MEPS-HC is representative of the civilian, noninstitutionalized population in the United States, the observational nature of the survey, as well as the cross-sectional analysis of the data, limits causal inferences. In addition, secondary analyses are limited by existing data. For example, we could obtain only the USC status and other items (ie, education, employment, insurance status) from parents residing in the respondent household. As with all studies that rely on self-report (or parental report), response bias is also a possibility. For example, a parent's report regarding his or her child's health care patterns may be biased because of the belief that a child's unmet needs reflect poorly on the parents. We did not measure type of USC or assess the comprehensive nature of the USC to determine whether the USC met the criteria needed to be considered a medical home. We did not investigate the type of health insurance or the effect that a specific type of plan may have had on receipt of health care services. Lastly, it is possible that parents do not have a USC because they do not believe that access to the health care system ensures health for their children or themselves and not because they cannot obtain one.

When parents lack a USC, their child typically receives fewer recommended health care services and experiences more unmet needs regardless of the child's USC and insurance status. Ensuring better access to necessary health care services will require comprehensive policy reforms that make it easier for all individuals to find and maintain a USC. Keeping the entire family in mind when crafting any new reforms will be essential to achieving a sustainable health care system and the best possible health outcomes for our children.

To read or post commentaries in response to this article, see it online at http://www.annfammed.org/content/9/6/504.

Key words: Usual source of care; access to health care; child health; health policy; primary care; continuity of care

Submitted March 8, 2011; submitted, revised, May 19, 2011; accepted June 22, 2011.

Funding support: This project was directly supported by grants 1 K08 HS16181 and 1 R01 HS018569 from the Agency for Healthcare Research and Quality (AHRQ) and the Oregon Health \& Science University Department of Medicine. This publication received indirect support from the Oregon Clinical and Translational Research Institute (OCTRI); grant number UL1 RR024140 from the National Center for Research Resources (NCRR), a component of the National Institutes of Health (NIH), and NIH Roadmap for Medical Research.

Acknowledgements: We wish to acknowledge the thousands of individuals who participated in the MEPS.

\section{References}

1. Gadomski A, Jenkins $P$, Nichols $M$. Impact of a Medicaid primary care provider and preventive care on pediatric hospitalization. Pediatrics. 1998;101(3):E1.

2. Starfield B. Evaluating the State Children's Health Insurance Program: critical considerations. Annu Rev Public Health. 2000;21:569-585.

3. Allred NJ, Wooten KG, Kong Y. The association of health insurance and continuous primary care in the medical home on vaccination coverage for 19- to 35-month-old children. Pediatrics. 2007;119 (Suppl 1):S4-S11.

4. Smith PJ, Santoli JM, Chu SY, Ochoa DQ, Rodewald LE. The association between having a medical home and vaccination coverage among children eligible for the vaccines for children program. Pediatrics. 2005;116(1):130-139.

5. Sox CM, Swartz K, Burstin HR, Brennan TA. Insurance or a regular physician: which is the most powerful predictor of health care? Am J Public Health. 1998;88(3):364-370.

6. Bartman BA, Moy E, D'Angelo LJ. Access to ambulatory care for adolescents: the role of a usual source of care. J Health Care Poor Underserved. 1997;8(2):214-226.

7. Bindman AB, Grumbach K, Osmond D, Vranizan K, Stewart AL. Primary care and receipt of preventive services. J Gen Intern Med. 1996;11(5):269-276. 
8. Piehl MD, Clemens CJ, Joines JD. "Narrowing the Gap": decreasing emergency department use by children enrolled in the Medicaid program by improving access to primary care. Arch Pediatr Adolesc Med. 2000;154(8):791-795.

9. Hoilette LK, Clark SJ, Gebremariam A, Davis MM. Usual source of care and unmet need among vulnerable children: 1998-2006. Pediatrics. 2009;123(2):e214-e219.

10. Starfield B, Shi L. The medical home, access to care, and insurance: a review of evidence. Pediatrics. 2004;113(5 Suppl):1493-1498.

11. DeVoe JE, Saultz JW, Krois L, Tillotson CJ. A medical home versus temporary housing: the importance of a stable usual source of care. Pediatrics. 2009;124(5):1363-1371.

12. Kaiser Commission on Medicaid and the Uninsured. Children's health-why insurance matters. The Henry J. Kaiser Family Foundation. http://www.kff.org/uninsured/4055-index.cfm. Accessed Aug 20, 2007.

13. Dubay L, Kenney GM. Health care access and use among low-income children: who fares best? Health Aff (Millwood). 2001;20(1):112-121.

14. Romaire MA, Bell JF. The medical home, preventive care screenings, and counseling for children: evidence from the Medical Expenditure Panel Survey. Acad Pediatr. 2010;10(5):338-345.

15. Ortega AN, Stewart DC, Dowshen SA, Katz SH. The impact of a pediatric medical home on immunization coverage. Clin Pediatr (Phila). 2000;39(2):89-96.

16. Cooley WC, McAllister JW, Sherrieb K, Kuhlthau K. Improved outcomes associated with medical home implementation in pediatric primary care. Pediatrics. 2009;124(1):358-364.

17. Overpeck MD, Kotch JB. The effect of US children's access to care on medical attention for injuries. Am J Public Health. 1995;85(3): 402-404.

18. Shipman SA, Lurie JD, Goodman DC. The general pediatrician: projecting future workforce supply and requirements. Pediatrics. 2004; 113(3 Pt 1):435-442.

19. Macinko JA, Shi L, Starfield B, Wulu JT Jr. Income inequality and health: a critical review of the literature. Med Care Res Rev. 2003; 60(4):407-452.

20. Guendelman S, Wier M, Angulo V, Oman D. The effects of childonly insurance coverage and family coverage on health care access and use: recent findings among low-income children in California. Health Serv Res. 2006;41(1):125-147.

21. Guendelman S, Pearl M. Children's ability to access and use health care. Health Aff (Millwood). 2004;23(2):235-244.

22. DeVoe JE, Tillotson CJ, Wallace LS. Children's receipt of health care services and family health insurance patterns. Ann Fam Med. 2009;7(5):406-413.

23. Davidoff A, Dubay L, Kenney G, Yemane A. The effect of parents' insurance coverage on access to care for low-income children. Inquiry. 2003;40(3):254-268.

24. Newacheck PW, Halfon N. The association between mother's and children's use of physician services. Med Care. 1986;24(1):30-38.

25. Minkovitz CS, O'Campo PJ, Chen YH, Grason HA. Associations between maternal and child health status and patterns of medical care use. Ambul Pediatr. 2002;2(2):85-92.

26. Weinick RM, Zuvekas SH, Drilea SK. Access to Health Care: Source and Barriers, 1996; MEPS Research Findings No. 3. AHCPR Pub. No. 98-0001. Rockville, MD: Agency for Health Care Policy and Research; 1997
27. Zuvekas SM, Weinick RM. Changes in access to care, 1977-1996: the role of health insurance. Health Serv Res. 1999;34(1, Part II): 271-279.

28. Cohen JW, Monheit AC, Beauregard KM, et al. The Medical Expenditure Panel Survey: a national health information resource. Inquiry. 1996-1997;33(4):373-389.

29. Cohen S. Sample Design of the 1996 Medical Expenditure Panel Survey Household Component. MEPS Methodology Report No. 2. Pub. No. 97-0027. Rockville, MD: Agency for Healthcare Research and Quality; 1997.

30. Agency for Healthcare Research and Quality. MEPS HC-089: 2004 full year consolidated data file. http://www.meps.ahrq.gov/mepsweb/ data_stats/download_data/pufs/h89/h89doc.pdf. Accessed Jan 10, 2008.

31. Newacheck PW, Hughes DC, Hung YY, Wong S, Stoddard JJ. The unmet health needs of America's children. Pediatrics. 2000;105 (4 Pt 2):989-997.

32. American Academy of Pediatrics. Recommendations for preventive pediatric health care (periodicity schedule). 2010. http://practice .aap.org/content. aspx?aid =1599\&nodeID $=4027$. Accessed Dec 8, 2010

33. American Academy of Pediatric Dentistry. Clinical Affairs Committee. Guideline on periodicity of examination, preventive dental services, anticipatory guidance/counseling, and oral treatment for infants, children, and adolescents. In: American Academy of Pediatric Dentistry 2010-11 Definitions, Oral Health Policies, and Clinical Guidelines. 2009;32(6)93-100. http://www.aapd.org/media/Policies _Guidelines/G_Periodicity.pdf.

34. Birken SA, Mayer ML. An investment in health: anticipating the cost of a usual source of care for children. Pediatrics. 2009;123(1):77-83.

35. Aday LA, Andersen R. A framework for the study of access to medical care. Health Serv Res. 1974;9(3):208-220.

36. DeVoe JE, Tillotson C, Wallace LS. Uninsured children and adolescents with insured parents. JAMA. 2008;300(16):1904-1913.

37. Department of Health and Human Services. Annual update of the HHS poverty guidelines. Fed Reg. 2007;72(15):3147-3148.

38. Bieler GS, Brown GG, Williams RL, Brogan DJ. Estimating modeladjusted risks, risk differences, and risk ratios from complex survey data. Am J Epidemiol. 2010;171(5):618-623.

39. DeVoe JE, Tillotson CJ, Wallace LS. Children's receipt of health care services and family health insurance patterns. Ann Fam Med. 2009;7(5):406-413.

40. DeVoe JE, Tillotson C, Lesko SE, Wallace LS, Angier H. The case for synergy between a usual source of care and health insurance coverage. J Gen Intern Med. 2011 March 16 [Epub ahead of print].

41. Devoe JE, Tillotson C, Wallace LS, Lesko SE, Angier H. Insurance and a usual source of care on a child's receipt of health care. J Pediatr Health Care. In press. http://www.jpedhc.org/article/ S0891-5245\%2811\%2900056-3/fulltext.

42. U.S. Department of Health and Human Services. Understanding the Affordable Care Act: timeline: what's changing and when. http:// www.healthcare.gov/law/timeline/index.html. Accessed Feb 1, 2011.

43. U.S. Department of Health and Human Services. Creating jobs and increasing the number of primary care providers. http://www. healthcare.gov/news/factsheets/creating_jobs_and_increasing_ primary_care_providers.html. Accessed May 18, 2011.

44. Vistnes JP, Schone BS. Pathways to coverage: the changing roles of public and private sources. Health Aff (Millwood). 2008;27(1):44-57. 